\title{
Neuroticism as a common dimension in the internalizing disorders
}

\author{
J. W. Griffith ${ }^{1 *}$, R. E. Zinbarg ${ }^{1}$, M. G. Craske ${ }^{2}$, S. Mineka ${ }^{1}$, R. D. Rose ${ }^{2}$, A. M. Waters ${ }^{3}$ and J. M. Sutton ${ }^{1}$ \\ ${ }^{1}$ Department of Psychology, Northwestern University, Chicago, IL, USA \\ ${ }^{2}$ Department of Psychology, University of California, Los Angeles, CA, USA \\ ${ }^{3}$ School of Applied Psychology, Griffith University, Gold Coast, Australia
}

Background. Several theories have posited a common internalizing factor to help account for the relationship between mood and anxiety disorders. These disorders are often co-morbid and strongly covary. Other theories and data suggest that personality traits may account, at least in part, for co-morbidity between depression and anxiety. The present study examined the relationship between neuroticism and an internalizing dimension common to mood and anxiety disorders.

Method. A sample of ethnically diverse adolescents $(n=621)$ completed self-report and peer-report measures of neuroticism. Participants also completed the Structured Clinical Interview for DSM-IV (SCID).

Results. Structural equation modeling showed that a single internalizing factor was common to lifetime diagnosis of mood and anxiety disorders, and this internalizing factor was strongly correlated with neuroticism. Neuroticism had a stronger correlation with an internalizing factor $(r=0.98)$ than with a substance use factor $(r=0.29)$. Therefore, neuroticism showed both convergent and discriminant validity.

Conclusions. These results provide further evidence that neuroticism is a necessary factor in structural theories of mood and anxiety disorders. In this study, the correlation between internalizing psychopathology and neuroticism approached 1.0, suggesting that neuroticism may be the core of internalizing psychopathology. Future studies are needed to examine this possibility in other populations, and to replicate our findings.

Received 14 December 2008; Revised 4 September 2009; Accepted 6 September 2009; First published online 11 November 2009

Key words : Anxiety disorders, co-morbidity, neuroticism, unipolar mood disorders.

\section{Introduction}

Co-morbidity among mental disorders has important implications for theories of psychopathology (e.g. Brown \& Barlow, 2002; Watson, 2005). ${ }^{1} \dagger$ Unipolar mood disorders and anxiety disorders are often comorbid, as shown in adolescent (Lewinsohn et al. 1997; Essau, 2003) and adult samples (e.g. Kessler et al. $2005 b) .^{2}$ Although many investigators have examined the relationships among mood and anxiety disorders (e.g. Krueger \& Markon, 2006), further work is needed to understand the common and specific elements of depression and anxiety. Anxiety and depression can be, in part, subsumed under internalizing psychopathology, which can be defined as the tendency to experience feelings or states that are inner-directed and usually accompanied by over-controlled behavior.

* Address for correspondence: Dr J. W. Griffith, Northwestern University, Feinberg School of Medicine, Department of Medical Social Sciences, Chicago, IL, USA.

(Email: jameswgriffith@gmail.com)

$\dagger$ The notes appear after the main text.
By contrast, externalizing psychopathology is the expression of under-controlled and maladaptive behaviors (Mash \& Dozois, 2003).

The structure of mental disorders is of theoretical and practical significance. The Diagnostic and Statistical Manual of Mental Disorders (DSM-IV-TR; APA, 2000) guides the assessment and treatment of psychopathology. However, the fundamental structure of DSM-IV-TR and its predecessors is problematic for several reasons, including high rates of co-morbidity (Watson, 2005). DSM-V is scheduled for release in 2012 (see http://www.psych.org/MainMenu/Research/ DSMIV/DSMV.aspx), and research on co-morbidity will inform its revision.

We have explored the co-morbidity of mood and anxiety disorders by examining some of their statistical and psychological commonalities. Although many theorists have posited that anxiety and depression share a common factor, we have examined the degree to which the personality trait of neuroticism represents that common factor in an adolescent sample. The current study fits into a broader perspective of the 
importance of linking personality traits to psychopathology (e.g. Watson et al. 1994; Widiger \& Smith, 2008).

\section{Neuroticism as a common factor}

Several theorists have posited that one factor is common to depression and anxiety, whereas others factors are more specific to depression than to anxiety and vice versa. Clark \& Watson (1991), in their tripartite model, posited that high negative affect (NA) was common to anxiety and depression, but low positive affect (PA) was specific to depression, and physiological hyperarousal was specific to anxiety. Mineka et al.'s (1998) integrative hierarchical model posited that high NA is a common factor in the mood and anxiety disorders, but that individual disorders contain relatively specific elements (see also Brown et al. 1998). Clark et al. (1994) argued that stable traits may also be common factors in anxiety and depression, and that trait NA (i.e. neuroticism) in particular may be a common vulnerability factor in anxiety and depression.

Several studies find one factor common among mood and anxiety disorders. Using the results of the National Comorbidity Survey (Kessler et al. 1994), Krueger (1999) found support for one internalizing psychopathology factor that was common to major depressive disorder, dysthymia, generalized anxiety disorder, social phobia, specific phobia, agoraphobia and panic disorder. The results of Krueger (1999) were replicated by Krueger \& Markon (2006) in a metaanalysis of five studies. In addition, McGlinchey \& Zimmerman (2007) examined psychiatric out-patients and found that one internalizing psychopathology factor was common to major depressive disorder, panic and agoraphobia, specific phobia, social phobia and generalized anxiety disorder. Finally, in a study of caretaker-reported psychopathology in twin children and adolescents, Lahey et al. (2008) found a higherorder internalizing factor that was common among mood and anxiety disorders.

Evidence that one factor is common to mood and anxiety disorders is consistent with models that posit the existence of a personality trait, namely neuroticism (a trait disposition to experience NA), that acts as a non-specific vulnerability factor to the internalizing disorders (e.g. Eysenck, 1967; Gray, 1982). Moreover, many studies have addressed the relationship between neuroticism and the dimension of internalizing psychopathology. For example, Brown et al. (1998) found that trait NA was common to depression and anxiety as measured by a combination of interview and questionnaires. Low trait PA was common to depression and social phobia, and autonomic arousal was related to panic and agoraphobia. Khan et al.
(2005) examined the population-based Virginia Twin Registry, which consisted of Caucasian adults, and found that neuroticism accounted for $20-45 \%$ of co-morbidity among various mood and anxiety disorders. Weinstock \& Whisman (2006) found that individuals with co-morbid mood and anxiety disorders scored higher on neuroticism than those individuals with either a mood disorder or an anxiety disorder alone. In turn, individuals with either a mood or an anxiety disorder scored higher on neuroticism than individuals with neither disorder. Kotov et al. (2007) examined neuroticism and other traits as they relate to anxiety symptoms; they found that neuroticism was associated with symptoms of social phobia, obsessivecompulsive disorder, panic disorder and generalized anxiety disorder. Krueger et al. (2001) also found an association between negative emotionality and internalizing psychopathology (in this study, internalizing psychopathology included major depression, panic disorder, social phobia and specific phobia). Thus, several studies demonstrate a relationship between neuroticism and mood and anxiety disorders.

Many studies of personality rely on self-report instruments, which are susceptible to bias and faking (Furnham, 1997). To address this issue, the current study included peer-report measures of neuroticism in addition to self-report measures. Achenbach et al. (2005) reviewed the literature and found that peerand self-report measures of internalizing and externalizing psychopathology correlated significantly, but that peer measures often had incremental validity. To the extent that peer measures yield different results than self-report measures, it suggests the importance of observable behavior, in addition to internal, cognitive processes.

\section{The current study}

We hypothesized that a shared internalizing disorder factor should be strongly related to neuroticism. To test this hypothesis, our study assessed neuroticism with self- and peer-report measures, and assessed mood and anxiety disorders using a semi-structured interview. We used an adolescent sample because adolescence is a developmental period characterized by important changes (Steinberg \& Morris, 2001), and because adolescence is a period of increased risk for mood and anxiety disorders. Indeed, many mental disorders begin during this time (e.g. Kessler et al. 2005a).

We also examined the discriminant validity or specificity of neuroticism to internalizing psychopathology. To examine discriminant validity, we analyzed substance use disorders, given that Krueger (1999) used substance use disorders as indicators of externalizing psychopathology. Although neuroticism 
is hypothesized to be related to internalizing psychopathology, Krueger et al. (1996) also found that individuals with diagnoses of substance dependence scored high on a measure of negative emotionality. Thus, we anticipated an association between substance use and neuroticism.

\section{Method}

\section{Participants}

Our data derive from a larger prospective study. Participants were students in their 11th year of education and were recruited over 3 years from one school in suburban Chicago, Illinois, and another in suburban Los Angeles, California. For screening, students provided assent and parental consent before completing the neuroticism scale of the revised Eysenck Personality Questionnaire (EPQ-R-N; Eysenck \& Eysenck, 1975). Over the 3 years, 1976 students completed the screening measure. Participants were paid US\$10 for the screening phase.

Students were categorized as low $(n=634)$, medium $(n=666)$ and high neuroticism $(n=676)$ based on the EPQ-R-N. ${ }^{3}$ Of these students, 1269 were invited into a longitudinal study. We oversampled high-neuroticism participants. Of those students invited into the study, 668 agreed to participate. Of these, 627 completed their baseline assessment, which included the Structured Clinical Interview for DSM-IV, non-patient edition (SCID-I/NP; First et al. 2002) and several questionnaires. Six cases were excluded from the present study because of missing data, or because of the possible presence of psychosis. The sample for the present study consisted of 114 low-neuroticism participants (18\%), 144 medium-neuroticism participants (23\%), and 363 high-neuroticism participants (59\%). Participants received US\$40 for completing the interview and questionnaires. The sample was $69 \%$ female, $48 \%$ Caucasian, $15 \%$ Latino, 13\% multi-ethnic, $13 \%$ African American, 5\% 'other', 4\% Asian, and 1\% Pacific Islander. At the time of their first interview, the sample ranged in age from 15 to 18 years $($ mean $=16.9$, S.D. $=0.4)$.

\section{Assessment procedures}

\section{Psychopathology}

Lifetime diagnoses based on the SCID-I/NP (hereafter referred to as the SCID) were the main outcome measures. We required that each disorder be characterized by clinically significant functional impairment (e.g. absenteeism) or clinically significant distress (e.g. pervasive distress about the symptoms, seeking treatment). The median interval between screening and the SCID was 3 months (range 1-15 months). Graduate students, research assistants or postdoctoral fellows were interviewers who were trained through self-study, didactics, role-playing, observations and tests of diagnostic ability. Interviewers were also trained to assess not otherwise specified (NOS) diagnoses. When an NOS diagnosis was assigned, interviewers noted which criteria set the symptoms most closely resembled. Interviewers presented assessments to doctoral-level supervisors at diagnostic meetings. The present study only includes analyses of clinically significant cases that met full criteria for one or more DSM syndromes.

To assess inter-rater reliability, trained interviewers observed SCIDs conducted by other interviewers for 69 cases. We assessed inter-rater reliability for diagnoses that occurred in three or more cases. $\kappa$ was acceptable in each case: major depressive disorder $(\kappa=0.83)$, social phobia $(\kappa=0.65)$, generalized anxiety disorder $(\kappa=$ $0.85)$, and obsessive-compulsive disorder $(\kappa=0.85)$

\section{Neuroticism: self-report}

Participants completed the EPQ-R-N, the Big-Five Mini-Markers Neuroticism scale (Big 5-N; Saucier, 1994), the broadband neuroticism scale from the International Personality Item Pool (IPIP-N; http:// ipip.ori.org/newNEOKey.htm, accessed 11 December 2007), and the Behavioral Inhibition System scale (BIS; Carver \& White, 1994). To better measure the general neuroticism factor and to eliminate redundancy, we dropped 25 of the 60 original items from the IPIP-N. The revision to the IPIP-N was based on a confirmatory factory analysis guided by Goldberg's (1999) facets. We retained or dropped items on the basis of factor loadings and item content. We dropped items with low factor loadings until omega $a_{\text {hierarchical }}\left(\omega_{\mathrm{h}}\right)$ began to decrease, and a final structural equation model indicated $\omega_{\mathrm{h}}$ was 0.81 (McDonald 1985, 1999; Zinbarg et al. 2005, 2006; Uliaszek et al. 2009). Coefficient $\omega_{\mathrm{h}}$ is the proportion of scale score variance accounted for by a general factor. For each neuroticism scale, Cronbach's $\alpha$ ranged from 0.75 to 0.93 . The EPQ$\mathrm{R}-\mathrm{N}$ has three lower-order facets; analyses of our screening data indicated that coefficient $\omega_{\mathrm{h}}$ is 0.68 for the EPQ-R-N (Mor et al. 2008).

We administered the EPQ-R-N during our screening phase. A packet of psychopathology, personality and cognitive style measures contained the other three neuroticism questionnaires. The median interval between the screening questionnaire and the administration of the questionnaire packet was 4 months (range 1-14 months).

Previous studies have shown that our neuroticism scales have adequate psychometric properties. Caruso 
Table 1. Descriptive statistics for lifetime diagnostic data

\begin{tabular}{|c|c|c|c|c|c|c|}
\hline & \multicolumn{3}{|c|}{ Percentage of sample } & \multicolumn{3}{|l|}{$n$} \\
\hline & Total & Male & Female & Total & Male & Female \\
\hline \multicolumn{7}{|l|}{ Mood disorders } \\
\hline Major depressive disorder & 20.0 & 13.9 & 22.7 & 124 & 27 & 97 \\
\hline Dysthymia & 1.3 & 1.0 & 1.4 & 8 & 2 & 6 \\
\hline \multicolumn{7}{|l|}{ Anxiety disorders } \\
\hline Social phobia & 9.2 & 11.9 & 8.0 & 57 & 23 & 34 \\
\hline Specific phobia: all subtypes & 6.4 & 3.1 & 8.0 & 40 & 6 & 34 \\
\hline Generalized anxiety disorder & 2.7 & 1.0 & 3.5 & 17 & 2 & 15 \\
\hline Obsessive compulsive disorder & 2.4 & 1.0 & 3.0 & 15 & 2 & 13 \\
\hline Panic disorder & 1.1 & 1.1 & 1.2 & 7 & 2 & 5 \\
\hline Post-traumatic stress disorder & 0.6 & 0.0 & 0.9 & 4 & 0 & 4 \\
\hline Acute stress disorder & 0.5 & 1.0 & 0.2 & 3 & 2 & 1 \\
\hline \multicolumn{7}{|l|}{ Substance use disorders } \\
\hline Alcohol abuse or dependence & 2.3 & 3.6 & 1.6 & 14 & 7 & 7 \\
\hline Cannabis abuse or dependence & 3.2 & 7.2 & 1.4 & 20 & 14 & 6 \\
\hline
\end{tabular}

et al. (2001) found that coefficient $\alpha$ for the EPQ-R-N ranged from 0.69 to 0.97 (median $=0.83$ ) across 69 samples. Muris et al. (2007) and Ross et al. (2002) found the BIS to have adequate reliability $(\alpha \geqslant 0.73)$. The BIS had convergent validity with our other measures of neuroticism ( $r$ 's ranged from 0.47 to 0.59 in our study). Saucier (1994) reported adequate reliability for the Big 5 -N scale $(\alpha=0.78)$, and also convergent validity with Goldberg's (1992) neuroticism scale. The neuroticism scales of the IPIP had adequate reliability ( $\alpha$ 's ranged from 0.77 to 0.88 ), and convergent validity with the NEO (validity coefficients ranged from 0.72 to 0.80 ; see http://ipip.ori.org/newBroadbandTable2.htm, accessed 11 December 2007).

\section{Neuroticism : peer report}

Each participant nominated two peers who knew them well. We only sought to obtain data from one peer, but obtained two contacts for cases in which the first peer did not participate. As in some other past studies (e.g. Marsh \& O'Neill, 1984), peers were asked to complete the questionnaires 'as if you were your friend who is completing the questionnaires about [themselves].' Each peer completed neuroticism measures: the Big 5-N, the IPIP-N and the BIS. They also completed a demographic questionnaire that assessed how long they had known the participant, frequency of contact, and confidence for their ratings. Internal consistency for peer measures ranged from 0.76 to 0.91. Descriptive statistics indicated that, on average, peers had known participants for 72 months (s.D. = 55), were in contact with the participant 5.3 days per week (s.D. =1.8), and had high levels of confidence in their ratings of participants' personalities (mean $=5.4$ on a 0-7 scale, s.D. $=1.3$ ). Peers received US\$15 for participating. Peer data were collected between 3 and 22 months (median $=6$ ) after participants completed questionnaires.

\section{Results \\ Data preparation}

We investigated threats to the accuracy of analyses by subjecting all variables to graphical and statistical analyses. For each self-report variable in the analysis, $<5 \%$ of cases had missing data. Peer data were available for slightly $>40 \%$ ( $n=258$ for IPIP-N and Big 5, and $n=253$ for the BIS) of the participants. We assumed that data in these analyses were missing at random. We used Mplus (Muthén \& Muthén, 19982007) for data analysis, which provides maximum likelihood estimates for parameters when data are missing at random. We followed recommendations by Winship \& Radbill (1994) and did not weight cases in most of our analyses. However, weighted analyses yielded almost identical results to unweighted analyses.

\section{Descriptive statistics}

Table 1 presents the diagnostic data. Descriptive statistics for neuroticism measures are presented in Table 2, and a correlation matrix for all variables is presented in Table 3. Among participants who had complete self-report data on at least three neuroticism scales $(n=599)$, participants with co-morbid anxiety 
Table 2. Descriptive statistics for self- and peer-report scales

\begin{tabular}{lllllcl}
\hline & $n$ & Mean & S.D. & Minimum & Maximum & Cronbach's $\alpha$ \\
\hline EPQ-R-N & 621 & 53.9 & 20.5 & 0.0 & 100.0 & 0.79 \\
Big 5-N & 593 & 46.9 & 17.7 & 6.3 & 95.3 & 0.80 \\
IPIP-N & 600 & 41.1 & 16.2 & 3.6 & 88.6 & 0.93 \\
BIS & 599 & 63.4 & 19.4 & 4.8 & 100.0 & 0.75 \\
Big 5-N-Peer & 255 & 48.4 & 19.4 & 3.1 & 93.8 & 0.83 \\
IPIP-N-Peer & 255 & 41.1 & 15.2 & 5.7 & 86.4 & 0.91 \\
BIS-Peer & 251 & 62.9 & 20.6 & 9.5 & 100.0 & 0.76 \\
\hline
\end{tabular}

EPQ-R-N, Revised Eysenck Personality Scale-Neuroticism; Big 5-N, Big 5 MiniMarkers-Neuroticism Scale; IPIP-N, International Personality Item Pool-Neuroticism Scale; BIS, Behavioral Inhibition System scale; S.D., standard deviation.

All variables were rescaled to the percentage of maximum possible scores (POMP; Cohen et al. 1999, 2003, p. 156). POMP scores can range from 0 to $100 \%$ and represent the percentage of the distance from the minimum to the maximum of a scale. The minimum and maximum scores presented are the empirical values from the sample; Cronbach's $\alpha$ was computed for participants analyzed in this study who had no missing items on the measure analyzed.

Table 3. Bivariate correlations for all variables

\begin{tabular}{|c|c|c|c|c|c|c|c|c|c|c|c|c|c|c|c|c|}
\hline & 1 & 2 & 3 & 4 & 5 & 6 & 7 & 8 & 9 & 10 & 11 & 12 & 13 & 14 & 15 & 16 \\
\hline \multicolumn{17}{|l|}{ 1. EPQ-R-N } \\
\hline 2. Big $5-\mathrm{N}$ & 0.50 & & & & & & & & & & & & & & & \\
\hline 3. IPIP-N & 0.60 & 0.69 & & & & & & & & & & & & & & \\
\hline 4. BIS & 0.47 & 0.51 & 0.59 & & & & & & & & & & & & & \\
\hline 5. Big 5-N-Peer & 0.25 & 0.29 & 0.24 & 0.16 & & & & & & & & & & & & \\
\hline 6. IPIP-N-Peer & 0.17 & 0.32 & 0.30 & 0.20 & 0.65 & & & & & & & & & & & \\
\hline 7. BIS-Peer & 0.14 & 0.25 & 0.24 & 0.27 & 0.42 & 0.53 & & & & & & & & & & \\
\hline 8. MDD & 0.40 & 0.24 & 0.34 & 0.24 & 0.12 & 0.15 & 0.11 & & & & & & & & & \\
\hline 9. Dysthymia & 0.45 & 0.13 & 0.18 & -0.08 & 0.23 & 0.15 & 0.03 & 0.33 & & & & & & & & \\
\hline 10. Panic disorder & 0.49 & 0.30 & 0.20 & 0.12 & -0.23 & -0.10 & -0.31 & 0.11 & 0.43 & & & & & & & \\
\hline 11. Social phobia & 0.50 & 0.42 & 0.52 & 0.38 & -0.02 & 0.16 & 0.08 & 0.44 & 0.52 & 0.10 & & & & & & \\
\hline 12. GAD & 0.51 & 0.38 & 0.49 & 0.47 & 0.20 & 0.17 & 0.22 & 0.21 & 0.32 & 0.35 & 0.43 & & & & & \\
\hline 13. Specific phobia & 0.22 & 0.28 & 0.27 & 0.19 & 0.45 & 0.23 & 0.17 & 0.34 & 0.11 & 0.38 & 0.35 & 0.15 & & & & \\
\hline 14. OCD & 0.35 & 0.26 & 0.32 & 0.37 & 0.41 & 0.25 & 0.02 & 0.46 & 0.30 & 0.37 & 0.31 & 0.19 & 0.30 & & & \\
\hline $\begin{array}{l}\text { 15. Alcohol-related } \\
\text { disorders }\end{array}$ & 0.38 & 0.32 & 0.31 & 0.26 & 0.00 & -0.02 & 0.06 & 0.28 & 0.31 & 0.34 & 0.42 & 0.18 & 0.32 & 0.23 & & \\
\hline $\begin{array}{l}\text { 16. Cannabis-related } \\
\text { disorders }\end{array}$ & 0.17 & 0.10 & 0.12 & 0.03 & 0.03 & 0.04 & -0.20 & 0.08 & 0.25 & 0.32 & 0.38 & 0.11 & -0.06 & 0.13 & 0.87 & \\
\hline
\end{tabular}

All correlations involving diagnostic variable are tetrachoric. EPQ-R-N, Revised Eysenck Personality Scale-Neuroticism; Big 5-N, Big 5 Mini-Markers-Neuroticism Scale; IPIP-N, International Personality Item Pool-Neuroticism Scale; BIS, Behavioral Inhibition System scale; MDD, major depressive disorder; GAD, generalized anxiety disorder; OCD, obsessive-compulsive disorder.

and depression scored higher on neuroticism than participants who had either an anxiety or a mood disorder. In turn, participants with either a mood or an anxiety disorder scored higher than participants with neither type of disorder $[F(2,596)=51.8, p<0.001$, Cohen's $d=0.7$ for both differences]. These results replicate those of Weinstock \& Whisman (2006).

\section{Measurement models}

We used categorical variables $(0=$ absence, $1=$ presence) to represent the lifetime prevalence of mood, anxiety and substance use disorders. The prevalence of substance use disorders was low in our sample, except for cannabis and alcohol use disorders. Thus, 
we only examined them and not other substance use disorders. To create substance use variables, we collapsed lifetime cannabis abuse and dependence into one variable, and collapsed lifetime alcohol abuse and dependence into another variable. Following some other studies, we excluded post-traumatic stress disorder $(n=4)$ and acute stress disorder $(n=3)$ because these diagnoses require an external event to occur. Thus, they are not good markers of an intrinsic dimension of internalizing psychopathology (Krueger, 1999).

To examine whether internalizing disorders loaded on one factor and substance use disorders loaded on another factor, we created two latent variables. Mood and anxiety disorders were loaded onto an internalizing psychopathology factor, and cannabis use and alcohol use disorders were loaded onto a substance use factor. The substance use and internalizing psychopathology factors were allowed to correlate. To identify our models, the loadings of the two substance use indicators were constrained to be equal. For this measurement model and all subsequent factor analyses, each diagnostic variable was regressed on its corresponding latent variable using probit regression assumptions. The variances of all latent factors were set to 1.0 to identify the models we tested.

The measurement model for the mood, anxiety and substance use disorders was an excellent fit as indicated by a comparative fit index of 0.99 (CFI; Bentler, 1988; Hu \& Bentler, 1999), a root mean square error of approximation of 0.01 (RMSEA; Browne \& Cudeck, 1993), and a weighted root-mean-square residual of 0.73 (WRMR; Muthén \& Muthén, 1998-2007; $\mathrm{Yu}, 2002)$. We also examined a measurement model for internalizing psychopathology alone. Mood and anxiety disorders were loaded on one latent factor. This model fit well $(\mathrm{CFI}=0.97$, RMSEA $=0.02, \mathrm{WRMR}=$ 0.71). Standardized factor loadings ranged from 0.44 to 0.73 . As we only had two indicators of substance use, we did not examine a measurement model.

To examine our measurement model for neuroticism, we created two latent factors that were allowed to correlate: neuroticism measured by self- and peerreport. This model was an excellent fit to the data $(\mathrm{CFI}=0.99$, RMSEA $=0.03$ with a $90 \%$ confidence interval of $0.00-0.06$; standardized root mean square residual $=0.03)^{4}$

\section{Neuroticism, internalizing disorders, and substance use disorders: structural models}

\section{Higher-order neuroticism factor}

We created a higher-order neuroticism factor indicated by the variables for self- and peer-reported neuroticism. To identify this model, we constrained the variance of the higher-order factor to 1.0. We also constrained the unstandardized paths from the higher-order factor to lower-order factors to 1.0. Because the substance use factor had two indicators, its variance was set to 1.0 and the loadings for the two indicators were constrained to be equal to identify the model. The higher-order neuroticism, internalizing psychopathology and substance use factors were allowed to correlate. This model provided an excellent fit to the data $(\mathrm{CFI}=0.95, \mathrm{RMSEA}=0.04, \mathrm{WRMR}=$ 0.97). The correlation between neuroticism and the internalizing psychopathology factor approached 1.0 (Fig. 1). This finding provides strong evidence of convergent validity for these two constructs. There was also a large, significant correlation between internalizing psychopathology and substance use $(r=0.45)$.

Because we oversampled high scorers on neuroticism, we also conducted a weighted analysis that gave less weight to high scorers on neuroticism. Using logistic regression, we analyzed the screened sample and modeled the probability of a participant entering the study as a function of the EPQ-R-N. Then, we weighted each case on the reciprocal of this probability. This approach gave more weight to cases that were less likely to be in our analyses. Because the correlation between internalizing psychopathology and neuroticism approached 1.0, we specified in Mplus that this correlation be $<0.99$. When we repeated our analysis in the weighted sample, we obtained very similar results with good fit indices $(\mathrm{CFI}=0.94$, $\mathrm{RMSEA}=0.03, \mathrm{WRMR}=0.94)$.

The correlation between neuroticism and substance use was smaller than the correlation between neuroticism and internalizing psychopathology (0.29 v. 0.98). A Wald test was used to determine whether the pathway from neuroticism to the internalizing psychopathology factor was different than the pathway from neuroticism to the substance use factor; this test was significant $\left[\chi^{2}(1)=20.3, p<0.0001\right]$. As predicted, higher-order neuroticism was more strongly related to internalizing disorders than to substance use disorders.

\section{Neuroticism and internalizing psychopathology: one and the same?}

Because the correlation between neuroticism and internalizing psychopathology approached 1.0, we investigated whether constraining the correlation between them as such would provide a good fitting model. As recommended by van der Sluis et al. (2005), we constrained the paths from internalizing psychopathology to substance use, and from neuroticism to substance use, to be equal. This model also provided 


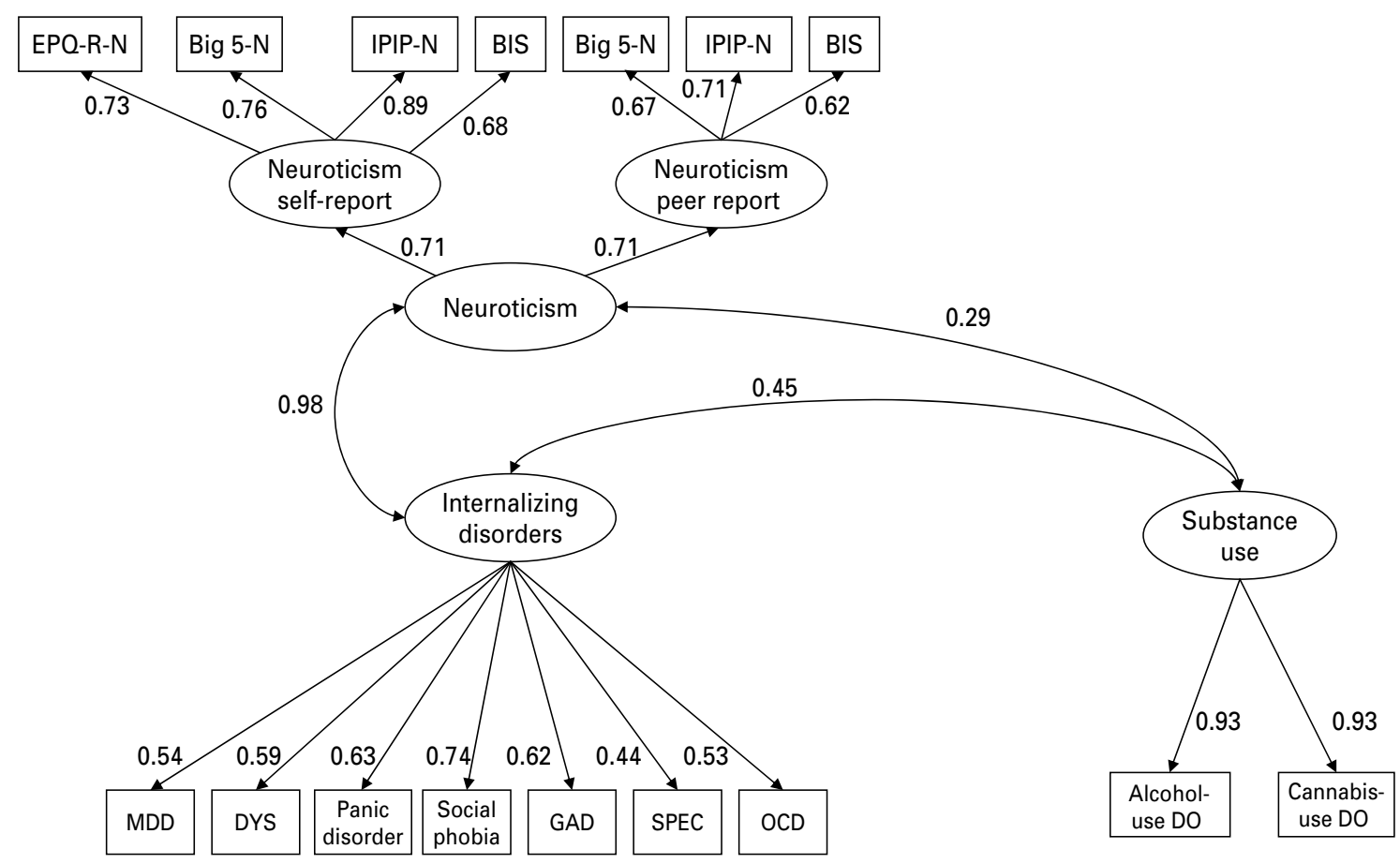

Fig. 1. Higher-order neuroticism, internalizing psychopathology, and substance use disorders. Arrows for error variance are omitted. All coefficients are standardized. MDD, major depressive disorder; DYS, dysthymia; GAD, generalized anxiety disorder; SPEC, specific phobia (all subtypes); OCD, obsessive-compulsive disorder; Alcohol-use DO, alcohol abuse or dependence; Cannabis-use DO, cannabis abuse or dependence; EPQ-R-N, Revised Eysenck Personality Scale-Neuroticism ; Big 5-N, Big 5 Mini-Markers Neuroticism Scale; IPIP-N, International Personality Item Pool-Neuroticism Scale; BIS, Behavioral Inhibition System scale. The numbers next to each path indicate standardized path coefficients or standardized factor loadings. All pathways are statistically significant at $p<0.05$. All but the pathway between neuroticism and substance use are significant at $p<0.01$.

an excellent fit to the data $(\mathrm{CFI}=0.95, \mathrm{RMSEA}=0.03$, $\mathrm{WRMR}=0.98$ ), and was not significantly different from the previous model without these constraints $\left[\chi^{2}(2)=1.62, p=0.45\right]$. Thus, a model that assumed a correlation of 1.0 between neuroticism and internalizing psychopathology was a good fit.

\section{Self- versus peer-reported neuroticism}

By eliminating the higher-order neuroticism factor, we examined whether peer-reported neuroticism differed from self-reported neuroticism (Fig. 2). We constrained the correlations between neuroticism and internalizing psychopathology, and also the correlations between neuroticism and substance use, to be equal for self- and peer-reported neuroticism. A Wald test showed that adding these two constraints would degrade the model $\left[\chi^{2}(2)=26.7, p<0.0001\right]$, indicating that self-reported neuroticism had different associations than peer-reported neuroticism. However, peer-reported neuroticism did share some variance with self-reported neuroticism $(r=0.42)$. The higher-order neuroticism factor, indicated by both self- and peer-report, had higher convergent validity

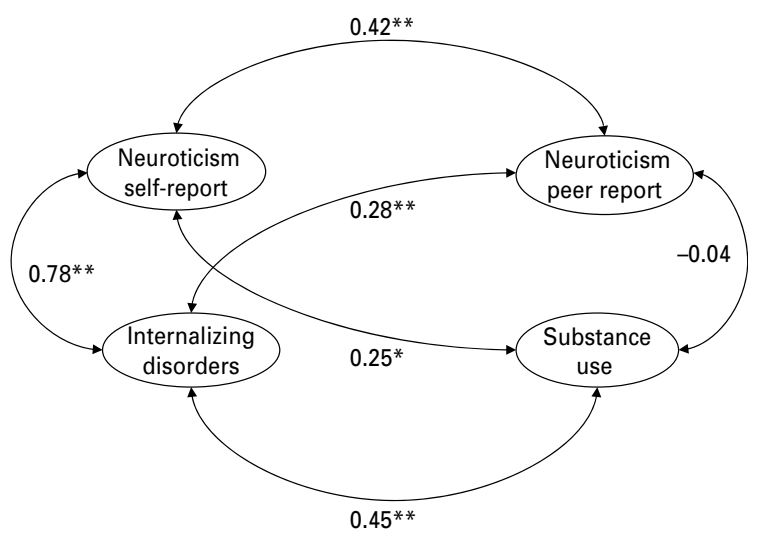

Fig. 2. Self- and peer-reported neuroticism, internalizing psychopathology, and substance use disorders. The measurement model is omitted. All coefficients are standardized. ${ }^{*} p<0.05,{ }^{* *} p \leqslant 0.01$.

than self-reported neuroticism alone with internalizing psychopathology (0.98 v. 0.78). However, discriminant validity coefficients with substance use were similar for high-order neuroticism and selfreported neuroticism (0.29 v. 0.25). In this model, 
internalizing psychopathology and substance use had a large, significant correlation $(r=0.45)$. We also analyzed this model with the weighted sample and obtained similar results $(\mathrm{CFI}=0.97$, RMSEA $=0.02$, $\mathrm{WRMR}=0.80$ ).

\section{Discussion}

We found that one internalizing psychopathology factor was strongly related to neuroticism. The relationship was so strong $(r=0.98)$ when neuroticism was indicated by self- and peer-report that it might be thought that the personality trait of neuroticism is the single characteristic common to anxiety and depression. However, such a strong conclusion would require replication of our study and more research across various populations. The results of the current study are suggestive, but not conclusive, that neuroticism and the core of internalizing psychopathology may be one and the same in adolescents. However, studies of adults (e.g. Krueger et al. 2001) have found smaller, yet significant, associations between internalization and neuroticism. Thus, neuroticism may be necessary to describe the structure of mood and anxiety disorders, but is not sufficient to describe all of the common variance across different populations. ${ }^{5}$

This study has two implications. First, we provide support for a common factor of neuroticism shared by anxiety and depression, as posited by Clark et al. (1994) and others. Therefore, our data support a structure of mood and anxiety disorders that differs from the structure implied by DSM-IV-TR. Second, our study suggests that, if investigators seek to measure variance common to depression and anxiety, they should use measures of neuroticism.

Our study had several important methodological features, including multiple self- and peer-reported measures of neuroticism. Peer-reported neuroticism had convergent and discriminant validity with internalizing psychopathology and substance use disorders. Moreover, peer measures increased the construct validity of neuroticism. The higher-order neuroticism factor, compared with the self-report factor, had higher convergent validity but similar discriminant validity. We showed a medium-to-large correlation between self- and peer-reported neuroticism, suggesting that there is common variance between self- and peer-report but also that substantial variance is unshared. Peer-report methodology can provide incremental information not available by self-report, and is less likely to be contaminated by certain biases. Therefore, peer-reported data should be considered for inclusion in future studies. Because self- and peer-reported neuroticism do not overlap completely, they may have different external correlates.

\section{Future research}

Although this study shows that neuroticism is common to mood and anxiety disorders, it is possible that studies that measure other traits in addition to neuroticism would yield different results. Future research should focus on other constructs that may be common to mood and anxiety disorders, including emotion regulation and rumination. Emotion regulation refers to biological and psychological processes that influence how emotions are expressed or experienced (Gross, 2002). It is possible that individual differences in emotion regulation predispose people to depression and anxiety, and therefore contribute to their covariation. Rumination is the tendency to think repetitively about negative emotions. NolenHoeksema (2000) found that rumination predicted symptoms of depression and anxiety, and also the onset of depressive episodes. Some researchers have examined neuroticism, depression and rumination in the same sample (e.g. Bagby \& Parker, 2001), but it is unclear whether rumination also predicts anxiety disorders and whether rumination is especially characteristic of individuals with co-morbid mood and anxiety disorders.

Future studies should examine the relationship between neuroticism and other forms of psychopathology, especially externalizing psychopathology. Several studies suggest relationships among externalizing psychopathology, internalizing psychopathology and neuroticism. For example, Krueger \& Markon (2006) found, in their meta-analysis, that internalizing psychopathology correlated 0.5 with externalizing psychopathology. In a study of child and adolescent twins, Lahey et al. (2008) found that major depressive disorder and generalized anxiety disorder were related strongly to both externalizing and internalizing symptoms. Sher et al. (2005) reviewed the literature and found that, in some studies, neuroticism seems to be an outcome of alcohol use, but other evidence suggests negative emotionality may precede problematic drinking. More research is needed, and the nature of the relationships among neuroticism, internalizing psychopathology and externalizing psychopathology can be clarified in longitudinal studies.

\section{Limitations}

We examined neuroticism, but did not examine other personality constructs. For example, Eysenck (1967, 1970) proposed that low extraversion also contributes to depression and anxiety. Although this proposal 
has received some empirical support (e.g. Trull \& Sher, 1994), other results suggest that the role of extraversion is weak when neuroticism is taken into account (e.g. Kendler et al. 2006).

Another limitation is that we oversampled participants who scored high on neuroticism. Consequently, the degree of covariation between mood and anxiety disorders may be higher than in an unselected sample. However, weighted analyses yielded similar results to unweighted analyses.

Another potential limitation in comparing our results to others in the literature is that we used an adolescent sample, whereas past studies have focused primarily on adults. Moreover, we used measures that are common in the adult literature, although the psychometric properties of neuroticism scales are not well established in adolescents. However, our measures had good psychometric properties in this sample. Nevertheless, further psychometric evaluation would be desirable.

Our study used the categorical approach of DSM-IV-TR, which has intrinsic limitations (e.g. Brown \& Barlow, 2002). We also combined current and past diagnoses to form diagnostic categories. This approach was the most practical because, in our sample, the rate of some current disorders was much lower than lifetime rates (e.g. current prevalence of mood disorders was only $6.6 \%$ ). However, it remains possible that the structure of mood and anxiety disorders might be different for a study examining only current diagnoses.

Finally, our sample did not have enough cases of substance use disorders to look beyond cannabis and alcohol use. However, the relationships among neuroticism, internalizing psychopathology and substance use disorders may be important for understanding co-morbidity. Neuroticism is related to some forms of alcohol dependence, but other forms of alcohol dependence may be more related to antisocial personality (Sher \& Trull, 1994; Sher et al. 2005). Thus, future studies that incorporate more aspects of personality, including personality disorders, may be better able to model the relationships among personality traits and substance use disorders.

The relatively few cases of substance use disorders also influenced our data analysis; we were forced to impose statistical constraints to empirically identify our models. Future studies with more cases of substance use disorders could extend our results by analyzing models without such statistical constraints. Elimination of these constraints and the inclusion of more externalizing psychopathology diagnostic categories (e.g. antisocial personality disorder, conduct disorder, other substance use disorders) would be important in future studies.

\section{Conclusions}

Previous work has shown strong covariation between mood and anxiety disorders that is consistent with one common internalizing psychopathology factor. Our data are consistent with the notion that the personality trait of neuroticism accounts, at least in part, for variance common across internalizing disorders. One possibility for future research is that neuroticism is the sole general factor in anxiety and depression (that is, is common to all anxiety and mood disorders). However, other studies that have examined neuroticism and internalizing psychopathology have found associations smaller than those found in the current study. For example, Krueger et al. (2001) found that negative emotionality and internalization correlated 0.27 in men and 0.22 in women. In contrast to the current study, participants in the Krueger et al. (2001) study were middle-aged, so it is possible that, with older age, other factors beyond neuroticism become increasingly important in mood and anxiety disorders. Future studies might examine how other factors, in addition to neuroticism, may be common to mood and anxiety disorders. In addition, specific factors in different forms of psychopathology need further exploration. Research examining anxiety and depression might include measures of neuroticism to help differentiate relationships between the common internalizing core from more specific aspects of depression and anxiety.

\section{Acknowledgments}

We thank the National Institutes of Health for supporting our research (Grant no. R01MH065652 to Drs Zinbarg and Mineka, and R01MH065651 to Dr Craske). We also acknowledge the assistance of numerous undergraduate and graduate students for their help in collecting and processing our data.

\section{Notes}

1 There is a debate among theorists regarding the appropriate use of the term co-morbidity. For example, Lilienfeld and colleagues (Lilienfeld et al. 1994; Lilienfeld, 2003) have argued that the term co-morbidity is misleading, is used inconsistently, and should be abandoned. Co-morbidity is used to describe diagnostic co-occurrence, and covariation between diagnoses across individuals. By contrast, Spitzer (1994) highlighted the merits of research on co-morbidity and argued that abandoning the term is unnecessary. In this paper, we use co-morbidity to refer to individuals who have been diagnosed with two or more mental disorders (i.e. co-occurrence of diagnoses). We use covariation in reference to statistical relationships between variables (psychodiagnostic and otherwise). 
${ }^{2}$ For brevity, we refer to unipolar mood disorders as mood disorders hereafter.

${ }^{3}$ The original neuroticism scale contains 24 items. We excluded the suicide item to reduce Institutional Review Board (IRB)-related concerns. We also excluded an item about health concerns from the analyses ('Do you worry about your health?'). In preliminary factor analyses, this item failed to load onto an overall neuroticism factor, and it did not load onto any lower-order factors (Mor et al. 2008).

${ }^{4}$ We also examined the scales for peer- and self-report separately and obtained excellent fitting models.

${ }^{5}$ In an examination of mood and anxiety disorders, Krueger (1999; Krueger \& Markon, 2006) found that internalizing psychopathology contains lower-order distress and fear factors, in addition to a higher-order internalization factor. Further study of neuroticism and internalizing psychopathology would be of interest at the level of lower-order factors. A single personality trait, such as neuroticism, may have different associations with lower-order factors, such as fear and distress factors. We attempted to replicate these lower-order factors of distress and fear, but were unsuccessful. This was not surprising given that our sample, although large by some standards, was much smaller than Krueger's (1999) and also in the meta-analytic study by Krueger \& Markon (2006). Although these lowerorder factors seem to be replicable, they are highly intercorrelated ( $r=0.74$ in Krueger \& Markon). Thus, even a model with two lower-order factors would strongly suggest one factor common to all of the internalizing disorders (i.e. a general factor). It was this general factor that was of central importance to our study.

\section{Declaration of Interest}

None.

\section{References}

Achenbach TM, Krukowski RA, Dumenci L, Ivanova MY (2005). Assessment of adult psychopathology: meta-analyses and implications of cross-informant correlations. Psychological Bulletin 131, 361-382. APA (2000). Diagnostic and Statistical Manual of Mental Disorders, 4th edn, text revision. American Psychiatric Association: Washington, DC.

Bagby RM, Parker JDA (2001). Relation of rumination and distraction with neuroticism and extraversion in a sample of patients with major depression. Cognitive Therapy and Research 25, 91-102.

Bentler PM (1988). Comparative fix indexes in structural models. Psychological Bulletin 107, 238-246.

Brown TA, Barlow DH (2002). Classification of mood and anxiety disorders. In Anxiety and its Disorders: The Nature and Treatment of Anxiety and Panic, 2nd edn (ed. D. H. Barlow), pp. 292-327. Guilford Press: New York.

Brown TA, Chorpita BF, Barlow DH (1998). Structural relationships among dimensions of the DSM-IV anxiety and mood disorders and dimensions of negative affect, positive affect, and autonomic arousal. Journal of Abnormal Psychology 107, 179-192.

Browne MW, Cudeck R (1993). Alternative ways of assessing model fit. In Testing Structural Models (ed. K. A. Bollen and J. S. Long), pp. 136-162. Sage Publications: Newbury Park, CA.

Caruso JC, Witkiewitz K, Belcourt-Dittloff A, Gottlieb J (2001). Reliability of scores from the Eysenck Personality Questionnaire: a reliability generalization study. Educational and Psychological Measurement 61, 675-689.

Carver CS, White TL (1994). Behavioral inhibition, behavioral activation, and affective responses to impending reward and punishment: the BIS/BAS scales. Journal of Personality and Social Psychology 67, 319-333.

Clark LA, Watson D (1991). Tripartite model of anxiety and depression: psychometric evidence and taxonomic implications. Journal of Abnormal Psychology 100, 316-336.

Clark LA, Watson D, Mineka S (1994). Temperament, personality, and the mood and anxiety disorders. Journal of Abnormal Psychology 103, 103-116.

Cohen P, Cohen J, Aiken LS, West SG (1999). The problem of units and the circumstance for POMP. Multivariate Behavioral Research 34, 315-346.

Cohen J, Cohen P, West SG, Aiken LS (2003). Applied Multiple Regression/Correlation Analysis for the Behavioral Sciences, 3rd edn. Lawrence Erlbaum Associates: Mahwah, NJ.

Essau CA (2003). Comorbidity of anxiety disorders in adolescents. Depression and Anxiety 18, 1-6.

Eysenck HJ (1967). The Biological Basis of Personality. Charles C. Thomas: Springfield, IL.

Eysenck HJ (1970). The Structure of Human Personality. Methuen and Co: London.

Eysenck HJ, Eysenck SBG (1975). Manual of the Eysenck Personality Questionnaire (Adult and Junior). Hodder \& Stoughton: London.

First MB, Spitzer RL, Gibbon M, Williams JBW (2002). Structured Clinical Interview for DSM-IV-TR Axis I Disorders, Research Version, Non-Patient Edition (SCID-I/NP).

Biometrics Research, New York State Psychiatric Institute: New York.

Furnham AF (1997). Knowing and faking one's five-factor personality score. Journal of Personality Assessment 69, 229-243.

Goldberg LR (1992). The development of markers for the Big-Five factor structure. Psychological Assessment 4, 26-42. Goldberg LR (1999). A broad-bandwidth, public-domain, personality inventory measuring the lower-level facets of several five-factor models. In Personality Psychology in Europe, vol. 7 (ed. I. Mervielde, I. Deary, F. De Fruyt and F. Ostendorf), pp. 7-28. Tilburg University Press: Tilburg, The Netherlands.

Gray JA (1982). A critique of Eysenck's theory of personality. In A Model for Personality (ed. H. J. Eysenck), pp. 246-276. Springer: New York.

Gross JJ (2002). Emotional regulation: affective, cognitive, and social consequences. Psychophysiology 39, 281-291. 
Hu L, Bentler PM (1999). Cutoff criteria for fit indices in covariance structure analysis: conventional criteria versus new alternatives. Structural Equation Modeling 6, 1-55.

Kendler KS, Gatz M, Gardner CO, Pederson NL (2006). Personality and major depression: a Swedish, population-based twin study. Archives of General Psychiatry 63, 1113-1120.

Kessler RC, Berglund P, Demler O, Jin R, Merikangas KR, Walters EE (2005a). Lifetime prevalence and age-of-onset distributions of DSM-IV disorders in the National Comorbidity Survey Replication. Archives of General Psychiatry 62, 593-602.

Kessler RC, Chiu WT, Demler O, Walters EE (2005b). Prevalence, severity, and comorbidity of the 12-month DSM-IV disorders in the National Comorbidity Survey Replication. Archives of General Psychiatry 62, 617-627.

Kessler RC, McGonagle KA, Zhao S, Nelson CB, Hughes M, Eshleman S, Wittchen H-U, Kendler KS (1994). Lifetime and 12-month prevalence of DSM-III-R psychiatric disorders in the United States. Archives of General Psychiatry 51, 8-19.

Khan AA, Jacobson KC, Gardner CO, Prescott CA, Kendler KS (2005). Personality and comorbidity of common psychiatric disorders. British Journal of Psychiatry 186, 190-196.

Kotov R, Watson D, Robles JP, Schmidt NB (2007). Personality traits and anxiety symptoms: the multilevel trait predictor model. Behaviour Research and Therapy 45, 1485-1503.

Krueger RF (1999). The structure of common mental disorders. Archives of General Psychiatry 56, 921-926.

Krueger RF, Caspi A, Moffitt TE, Silva PA, McGee R (1996). Personality traits are differentially linked to mental disorders: a multitrait-multidiagnosis study of an adolescent birth cohort. Journal of Abnormal Psychology 105, 299-312.

Krueger RF, Markon KE (2006). Reinterpreting comorbidity: a model-based approach to understanding and classifying psychopathology. Annual Review of Clinical Psychology 2, 111-133.

Krueger RF, McGue M, Iacono WG (2001). The higher-order structure of common DSM mental disorders: internalization, externalization, and their connections to personality. Personality and Individual Differences 30, 1245-1259.

Lahey BB, Rathouz PJ, Van Hulle C, Urbano RC, Krueger RF, Applegate B, Garriock HA, Chapman DA, Waldman ID (2008). Testing structural models of DSM-IV symptoms of common forms of child and adolescent psychopathology. Journal of Abnormal Child Psychology 36, 187-206.

Lewinsohn PM, Zinbarg R, Seeley JR, Lewinsohn M, Sack WH (1997). Lifetime comorbidity among anxiety disorders and between anxiety disorders and other mental disorders in adolescents. Journal of Anxiety Disorders 11, 377-394.

Lilienfeld SO (2003). Comorbidity between and within childhood externalizing and internalizing disorders: reflections and directions. Journal of Abnormal Child Psychology 31, 285-291.
Lilienfeld SO, Waldman ID, Israel AC (1994). A critical examination of the term and concept of 'comorbidity' in psychopathology research. Clinical Psychology: Science and Practice 1, 71-83.

Mash EJ, Dozois DJ (2003). Child psychopathology: a developmental systems perspective. In Child Psychopathology, 2nd edn (ed. E. J. Mash and R. A. Barkley), pp. 3-71. Guilford Press: New York.

Marsh HW, O'Neill R (1984). Self-description questionnaire III: The construct validity of multidimensional self-concept ratings by late adolescents. Journal of Educational Measurement 21, 153-174.

McDonald RP (1985). Factor Analysis and Related Methods. Erlbaum: Hillsdale, NJ.

McDonald RP (1999). Test Theory: A Unified Treatment. Erlbaum: Mahwah, NJ.

McGlinchey JB, Zimmerman M (2007). Examining a dimensional representation of depression and anxiety disorders' comorbidity in psychiatric outpatients with item response modeling. Journal of Abnormal Psychology 116, 464-474.

Mineka S, Watson D, Clark LA (1998). Comorbidity of anxiety and unipolar mood disorders. Annual Review of Psychology 49, 377-412.

Mor N, Zinbarg RE, Craske MG, Mineka S, Uliaszek A, Rose R, Griffith JW, Waters AM (2008). Evaluating the invariance of the factor structure of the EPQ-R-N among adolescents. Journal of Research in Personality $\mathbf{1}$, 66-75.

Muris P, Rassin E, Franken I, Leemreis W (2007). Psychometric properties of the behavioral inhibition scale in young adults. Journal of Individual Differences 28, 219-226.

Muthén LK, Muthén BO (1998-2007). Mplus User's Guide, 5th edn. Muthén \& Muthén: Los Angeles.

Nolen-Hoeksema S (2000). The role of rumination in depressive disorders and mixed anxiety/depressive symptoms. Journal of Abnormal Psychology 109, 504-511.

Ross SR, Millis SR, Bonebright TL, Bailey SE (2002). Confirmatory factor analysis of the Behavioral Inhibition and Activation Scales. Personality and Individual Differences 33, 861-865.

Saucier G (1994). Mini-markers: a brief version of Goldberg's unipolar big-five markers. Journal of Personality Assessment 63, 506-516.

Sher KJ, Grekin ER, Williams NA (2005). The development of alcohol use disorders. Annual Review of Clinical Psychology 1, 493-523.

Sher KJ, Trull TJ (1994). Personality and disinhibitory psychopathology: alcoholism and antisocial personality disorder. Journal of Abnormal Psychology 103, 92-102.

Spitzer RL (1994). Psychiatric 'Co-occurrence' ? I'll stick with 'Comorbidity'. Clinical Psychology: Science and Practice 1, 88-92.

Steinberg L, Morris AS (2001). Adolescent development. Annual Review of Psychology 52, 83-110.

Trull TJ, Sher KJ (1994). Relationship between the five-factor model of personality and Axis I disorders in a nonclinical sample. Journal of Abnormal Psychology 103, 350-360. 
Uliaszek A, Hauner K, Zinbarg RE, Mineka S, Craske MG, Griffith JW, Rose R (2009). An examination of content overlap and disorder-specific predictions in the associations of neuroticism with anxiety and depression. Journal of Research in Personality 43, 785-794.

van der Sluis S, Dolan CV, Stoel RD (2005). A note on testing perfect correlations in SEM. Structural Equation Modeling 12, 551-577.

Watson D (2005). Rethinking the mood and anxiety disorders: a quantitative hierarchical model for DSM-V. Journal of Abnormal Psychology 114, 522-536.

Watson D, Clark LA, Harkness AR (1994). Structures of personality and their relevance to psychopathology. Journal of Abnormal Psychology 103, 18-31.

Weinstock LM, Whisman MA (2006). Neuroticism as a common feature of the depressive and anxiety disorders: a test of the revised integrative hierarchical model in a national sample. Journal of Abnormal Psychology $115,68-74$.
Widiger TA, Smith GT (2008). Personality and psychopathology. In Handbook of Personality, 3rd edn (ed. O. P. John, R. W. Robins and L. A. Pervin), pp. 743-769. Guilford Press: New York.

Winship C, Radbill L (1994). Sampling weights and regression analyses. Sociological Methods and Research 23, 230-257.

Yu CY (2002). Evaluating Cutoff Criteria of Model Fit Indices for Latent Variable Models with Binary and Continuous Outcomes. Doctoral dissertation, University of California, Los Angeles.

Zinbarg RE, Revelle W, Yovel I, Li W (2005). Cronbach's $\alpha$, Revelle's $\beta$, and McDonald's $\omega_{\mathrm{H}}$ : their relationships with each other and two alternative conceptualizations of reliability. Psychometrika 70, 1-11.

Zinbarg RE, Yovel I, Revelle W, McDonald RP (2006). Estimating generalizability to a latent variable common to all of a scale's indicators: a comparison of estimators for $\omega_{\mathrm{h}}$. Applied Psychological Measurement 30, 121-144. 